\title{
Heterochirality results from reduction of maternal diaph expression in a terrestrial pulmonate snail
}

\author{
Takeshi Noda $^{1^{*}} \mathbb{D}$, Noriyuki Satoh ${ }^{2}$ and Takahiro Asami ${ }^{*}$
}

\begin{abstract}
Background: Spiral cleavage is a feature of non-ecdysozoan protostomes, in which left-right reversal frequently evolved in gastropod molluscs. In pulmonate gastropods, maternal molecules are responsible for chirality patterning, on which the polarities of visceral and coiling asymmetries depend. In the pond snail, Lymnaea stagnalis (the clade Hygrophila), a frame-shift mutation of one of tandem-duplicated, diaphanous-related formin genes (diaph) resulted in incomplete reversal from dextral to sinistral cleavage. Is this mechanism of chirality regulation common to, or shared with other pulmonates? To answer this question, we examined genes involved in chirality patterning in the land snail, Bradybaena similaris which belongs to the clade Stylommatophora.

Results: Both dextral and sinistral siblings develop from progeny of a racemic mutant of $B$. similaris. Differences in maternal mRNAs between the two strains were searched by transcriptome analyses. We found fifty maternal transcripts that exhibited less expression in early embryos of the mutant strain. The most conspicuous was a homolog of diaph. The diaph gene was duplicated in the stylommatophoran ancestor (diaph-a and diaph-b), as in the case of the ancestor of Lymnaea (Lsdiaph1 and Lsdiaph2). The quantity of maternal diaph-b mRNA was drastically reduced in early embryos of the racemic mutant compared to wild-type, while diaph-a expression was at nearly the same level in both strains. Unlike the case of Lsdiaph2, which is frame-shifted to produce truncated peptides in the mutant of L. stagnalis, however, Bsdiaph-b mRNA in the mutant strain is not frame-shifted and most probably produces normal Diaph-b protein. These results suggest the presence of regulatory mechanisms of gene expression for chirality patterning in pulmonate gastropods, although genomic analyses are required for confirmation.

Conclusions: Heterochirality resulting from the loss of polarity control in spiral cleavage does not require mutation of the diaph gene in B. similaris. The determination of left-right polarity instead depends on the expression of this diaph gene, which is duplicated in stylommatophoran Bradybaena, as well as in hygrophilan Lymnaea. Our results provide an avenue to identifying a regulatory mechanism that controls the direction of spiral cleavage in gastropods.
\end{abstract}

Keywords: Spiral cleavage, Left-right asymmetry, Gene duplication, Bradybaena, Pulmonate, Gastropod

\footnotetext{
*Correspondence: takeshinoda0@gmail.com; asami99@shinshu-u.ac.jp

'Department of Biology, Faculty of Sciences, Shinshu University, Matsumoto

390-8621, Japan

Full list of author information is available at the end of the article
}

(c) The Author(s). 2019 Open Access This article is distributed under the terms of the Creative Commons Attribution 4.0 International License (http://creativecommons.org/licenses/by/4.0/), which permits unrestricted use, distribution, and reproduction in any medium, provided you give appropriate credit to the original author(s) and the source, provide a link to the Creative Commons license, and indicate if changes were made. The Creative Commons Public Domain Dedication waiver (http://creativecommons.org/publicdomain/zero/1.0/) applies to the data made available in this article, unless otherwise stated. 


\section{Background}

Metazoans exhibit different modes of cleavage, including radial (starfish), spiral (snails), and bilateral (tunicates) cleavage (e.g., [1] Gilbert and Raunio, 1997; [2] Nielsen, 2012). Spiral cleavage is a characteristic for several taxa of non-ecdysozoan protostomes, represented by molluscs and annelids, and is recognized as an embryological feature of these animal groups (lophotrochozoans or spiralians), although the "Spiralia" vs "Lophotrochozoa" nomenclature has been debated (e.g., [3] Dunn et al., 2008 [4] Henry, 2014; [5] Laumer et al., 2015). In gastropod molluscs, the left-right polarity of spiral cleavage makes the blastomere asymmetric. The left-right geometry of blastomeres by itself regulates the handedness of subsequent zygotic gene expression, so as to develop into a clockwise-coiled (dextral) or counterclockwise-coiled (sinistral) snail ([6] Kuroda et al., 2009).

Maternal inheritance ([7] Toyama, 1913) of left-right reversal by nuclear gene mutation in pulmonates, which are hermaphrodites, was discovered through a breeding experiment with the dextral pond snail Lymnaea peregra ([8] Boycott and Driver, 1923, [9] Sturtevant, 1923). Mutant hatchlings exhibited reversal in bilateral visceral asymmetry as well as coiling direction. Transplantation experiments of egg cytoplasm between the wild-type dextral and mutant sinistral strains of $L$. peregra demonstrated the presence of maternally supplied information that is involved in patterning of the spiral cleavage ([10] Freeman and Lundelius, 1982). In L. stagnalis, progeny of a wild-type mother ( $D D$ or $D s)$ exhibit dextral cleavage while those of a mutant $(s s)$ display incomplete sinistral cleavage ([11] Asami et al., 2008, [12] Utsuno et al. 2011). In efforts to discover the maternally expressed genes responsible for patterning the chirality ([13] Hierck et al., 2005, [14] Shibazaki et al., 2004, [15] Harada et al., 2004), Davison et al. [16] and Kuroda et al. [17] independently succeeded in identifying diaphanous-related formin (the homolog of human diaph1, diaph2, and diaph3) as a candidate gene associated with the direction of left-right asymmetry in L. stagnalis. In this species, diaph is duplicated into two copies, one of which became frame-shifted in mutants. Viable progeny of the mutant homozygote (ss) develop into the sinistral form, while most siblings fail to develop ([12] Utsuno et al. 2011). Thus, these maternally expressed genes may be responsible for symmetry breaking in the Hygrophila, which is one of the two clades dividing the Pulmonata.

Left-right reversed groups/species have frequently evolved in gastropods, especially in pulmonates, among the Lophotrochozoa/Spiralia ([18] Okumura et al., 2008, [19] Gittenberger et al., 2012). In each phylogenetically independent event, a breeding population must have been fixed for a normally viable mutant allele for reversal. For example, in the Stylommatophora, the sister clade to the Hygrophila, populations repeatedly fixed for reversal in Euhadra ([20] Uesima and Asami, 2003) and Satsuma ([21] Hoso et al. 2010) of the mainly dextral Camaenidae as well as in Albinaria of the mainly sinistral Clausiliidae ([22] Kornilios et al., 2015). Within the Hygrophila, diaph is duplicated in the dextral Lymnaea, but not in the sinistral sister groups, Physella acuta or Indoplanorbis exustuse ([16] Davison et al., 2016, Noda et al., unpublished data). The commonality and diversity of genic mechanisms responsible for these recurrent evolutions of reversal remain unclear.

The racemic mutant of the dextral land snail Bradybaena similaris produces both dextral and sinistral progeny. This racemism provides a unique opportunity to examine the genic mechanism for the regulation of left-right polarity of spiral cleavage in the Camaenidae. A reverse-coiled mutant can rarely reproduce because of physical difficulty in mating with the wild type even if it was found in the wild, especially in case of simultaneously reciprocally copulating stylommatophorans such as camaenids ([20] Ueshima and Asami, 2003; [23] Asami et al. 1998). We however discovered a wild individual that was phenotypically dextral, but homozygous for the racemic allele, which made it possible to establish a racemic strain of B. similaris ([24] Utsuno and Asami, 2010). The mutant strains show maternal inheritance for chirality with genetically recessive alleles, which relies on maternal storage of a gene product that promotes dextral cleavage in the wild type. The racemism suggests that this phenotype may results from a loss-of-function mutation for determination of left-right polarity in spiral cleavage. By taking advantage of this chirality-mutant strain, only available in the Stylommatophora, we carried out RNA-seq analyses to test whether the racemic mutant exhibits a transcriptional defect.

Here we show that the maternal expression of one of the diaph-homologs is conspicuously reduced in the progeny of the racemic mutant of $B$. similaris with no mutation in the gene itself. Our results open a new ground to explore a regulatory mechanism that controls the left-right polarity of development in gastropods.

\section{Materials and methods Biological materials}

The land snail Bradybaena similaris, was used in this study. Snails of the dextral wild type and racemic mutant were collected from Kashiwa, Chiba, Japan and have been maintained in our laboratory ([24] Utsuno and Asami, 2010). Wild-type snails exhibit the clockwise-coiled (dextral) phenotype (Fig. 1A-a) whereas the progeny of the racemic mutant includes both clockwise- and counterclockwise-coiled (sinistral) siblings (Fig. 1A-b). Snails of the Pulmonata, including $B$. 
A
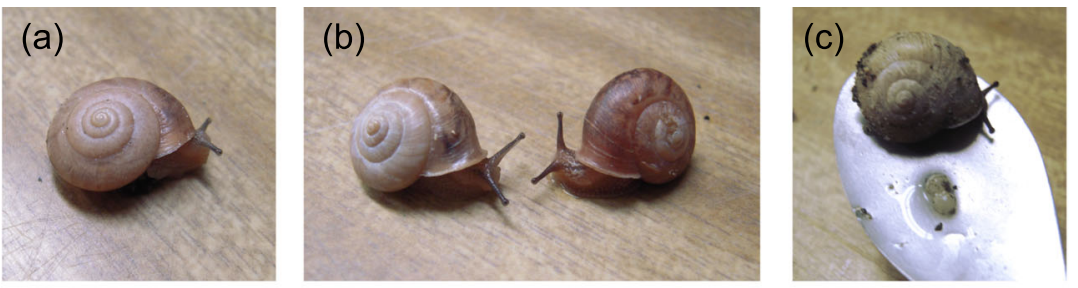

B
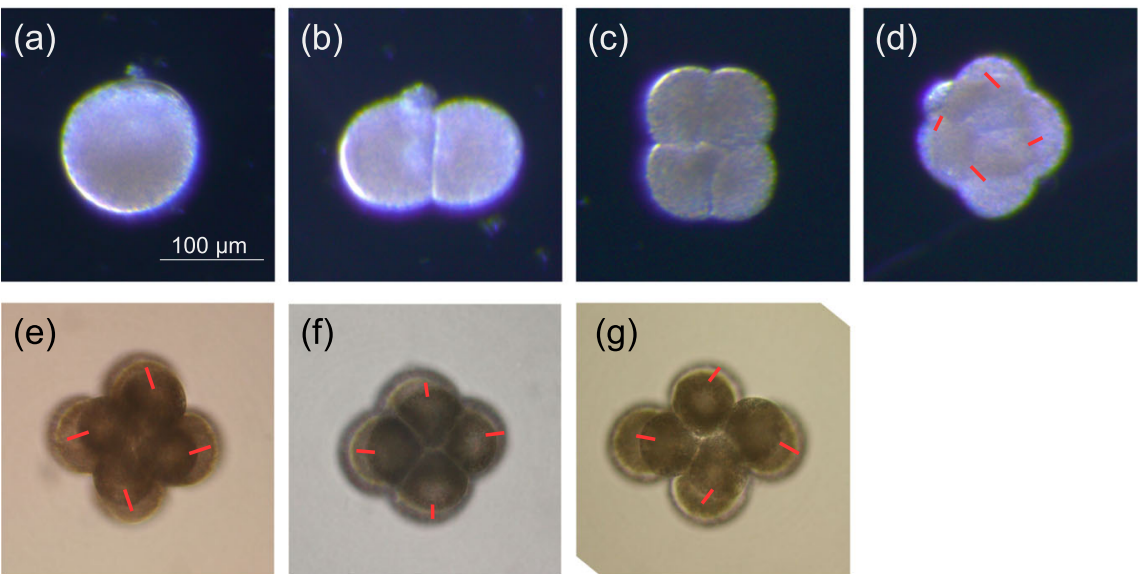

Fig. 1 (a) Adults of Bradybaena similaris. (a) Wild B. similaris (dextral). (b) Racemic mutants of B. similaris. Dextral (left) and sinistral (right) ones. Wild-type strain that produces clockwise-coiled (dextral) snails and a mutant strain that produces either clockwise or counterclockwise-coiled (sinistral) snails (right). (c) B. similaris lays several tens eggs during a period of several hours. (b) Embryos of B. similaris. (a)-(d) Embryos from a wild individual. (e)-(g) Embryos from a racemic mutant individual. (a) egg, (b) 2-cell, (c) 4-cell, (d)-(g) 8-cell. Cleavage directions are shown by red lines. Cells divide clockwise direction (d) in wild 4-cell embryos from animal pole view, while clockwise to counterclockwise direction (e)-(g) in racemic mutant ones

similaris, are hermaphroditic. Their fertilization occurs internally through simultaneous reciprocal copulation with the other individual with the same chiral phenotype.

The direction of spiral cleavage is determined maternally. Genetic studies showed that offspring of a ++ or + $r$ mother all become dextral individuals. On the other hand, $r r$ homozygosity has variable effects on embryogenesis, and usually result in 70\% dextral and 30\% sinistral offspring ([24] Utsuno and Asami, 2010). Females usually lay several tens of eggs over a period of several hours (Fig. 1A-c). This egg laying behavior makes difficult to collect enough eggs before the initiation of cleavage. We sampled early-embryos from the 1-cell to 16-cell stages for analysis of maternal genes. Although it is not known when maternal to zygotic transition initiates in B. similaris, 16-cell stage is the first stage for transcription in another gastropod species, Lymnaea([25] Biggelaar, 1971). Two sets of RNA-seq analyses were carried out using approximately 100 early embryos each from six to eight clutches. In addition, juveniles and digestive glands of adults were collected to examine their gene expression profiles.
RNA-seq, analyses of differential gene expression, mRNA quantification and molecular phylogeny

Total RNA was extracted using a standard TRIzol protocol procedure (Thermo Fisher Scientific), and cDNA libraries were prepared using a TruSeq RNA Library Prep Kit v2 (Illumina). RNA quality was checked with an Agilent Technologies 2100 Bioanalyzer using an Agilent RNA 6000 Nano Kit. Sequencing was performed using an Illumina Hiseq4000 and MiSeq. De novo assembly of whole RNA sequence reads was performed using a de Bruijn graph-based program, Trinity-v2.3.2 ([26] Grabherr et al., 2011; [27] Haas et al., 2013). All Illumina reads are available from NCBI database under accession nos. SRR804510-SRR804517.

Quantification of transcripts was carried out by software tools for expression analysis, Tophat 2.1.1 and Cufflinks 2.2.1([28] Trapnell et al., 2012) using RNA-seq results and the gene models constructed by Trinity. Differentially expressed gene analysis employed EdgeR 3.22 .5 ([29] Robinson et al., 2010) with the mapping results from Tophat. Differentially expressed genes were annotated using Blastx against reference protein sequences of Homo sapiens and genome sequences 
decoded in the mollusc, Biomphalaria glabrata, in the NCBI database. Quantification by qRT-PCR was also carried out as previously described with slight modifications ([30] Noda, 2011). Total RNA from batches of early embryos (egg to 16-cell stage) was extracted by standard TRIzol protocol. Three each of samples were prepared from siblings of wild, racemic and F1 strains. cDNA reverse transcribed from $10 \mathrm{ng}$ total RNA was used for each qRT-PCR reaction with step one plus real time PCR system (step one software version 2.3) by the standard curve methods. Sequences of primers are as follows; Bsidiaph- $b$ forword; TCAAAGACTGTGAT TGGCTGA, Bsidiaph- $b$ reverse; GCTCAGAGAATTCA TGAGTACCG, Bsidiaph-a forword; CCATGAAGC TTCCGTTTGAT, Bsidiaph-a reverse; TTCATGTCA TCTGGCTCTGG. We quantified all splicing variants of Bsdiaph-a together because of technical difficulty to distinguish Bsdiaph- $a-\times 1$ and Bsdiaph- $a-\times 2$ for quantification, including the construction of specific primers to each variant for qRT-PCR.

Molecular phylogenetic analysis was carried out with MrBayes version 3.2.6, as previously described with slight modifications ([30] Noda, 2011). Amino acid sequences of conserved $\mathrm{FH} 2$ domains were aligned by ClustalW version 2.1.0 for constructing the molecular phylogenetic tree. LG model was adopted as an evolutionary model. List of sequence IDs are available in Additional file 1: Table S2.

All informatic analyses were carried out using default parameters.

\section{Results}

Identification of differentially-expressed maternal genes in wild and mutant strains

Total RNA-seq reads obtained for wild-1embryos, wild-2 embryos, mutant- 1 embryos, mutant- 2 embryos, wild juveniles, and mutant juveniles, wild digestive gland, and mutant digestive gland were 24,192,382, 27,239,716, 30,957,316, 39,046,157, 29,971,051, 36,976,111, 30,075,674, and $31,158,448$, respectively (Additional file 2). After Trinity treatment, reads were assembled to 590,901 contigs (Additional file 2). The N50 of the assembled transcriptomes was 829 nucleotides (nts) (the longest being 31,291 nts). RNA-seq reads were mapped to these contigs using Tophat for the differentially expressed gene analysis and mRNA quantification.

Comparisons using the EdgeR method ([29] Robinson et al., 2010; [31] McCarthy et al., 2012) of the maternal transcriptomes between wild-type and mutant strains $(p$-value $<1 \mathrm{e}-30)$ demonstrated 50 genes that were significantly higher in the former than in the latter (Table 1). BlastX search against the Homo sapiens genome and that of a gastropod, Biomphalaria glabrata, was carried out to determine the similarity of transcripts to known proteins listed in the NCBI database. Of those,
34 transcripts showed similarity to proteins within the database, while another 16 showed no sequence similarity (Table 1). The transcript that was most reduced in mutant strains encodes a homolog of human DIAPH2 (Table 1, TRINITY_DN171781_c1_g2, $p$-value from EdgeR; 7.34E-158, LogFc; 6.26). So, we named the gene as Bsdiaph (B. similaris gene for Diaph protein). The reads of Bsdiaph transcript were abundant in early embryos of the wild-type (3933 reads in Exp-1 and 4207 in Exp-2) and very few in early embryos of mutants (49 reads in Exp-1 and 57 in Exp-2). This result suggests that maternal expression of diaph is greatly suppressed in the B. similaris mutant strain, in which embryogenesis produced racemic (dextral and sinistral) progeny.

The next four transcripts with sequence similarity to human proteins corresponded to RIMS2 (TRINITY_DN179541_c2 g2), POL1 (TRINITY_DN15823 6_c0_g1), TDRD15 (TRINITY_DN174403_c0_g1) and CYP17A1 (TRINITY_DN169314_c0_g5_i2) (Table 1).

TRINITY_DN158236_c0_g1 is an orthologue of human POLI, which is an error-prone DNA polymerase involved in DNA repair. Genomes of Homo sapiens, Biomophalalia glablata and Aplysia californica and our transcriptome data contain each one POLI orthologue. TRINITY_DN184104_c10_g9 is a homologue of human CYP17A1, which is a member of the cytochrome P450 superfamily of enzymes for metabolic reactions, however, orthologous relationship with human genes was not clear because of highly diversification of these genes. Genomes of Biomophalalia glablata and Aplysia californica contain each one orthologue of TRINITY_DN184104_c10_g9. The homology between human RIMS2 and TDRD15 and Bradybaena transcripts (TRINITY_DN179541_c2_g2 and TRINITY_DN174403_c0_g1) was not clear, because their similarity was limited to small protein motifs or domains. These genes were highly diversified or independent in the Pulmonata lineage. Further characterization of genes with weak expression in racemic mutant embryos is the focus of our next investigation.

\section{Identification and characterization of diaph- $a$ and diaph- $b$ transcripts in Bradybaena similaris}

In L. stagnalis, diaph duplicated into Lsdiaph1 and Lsdiaph2, which are tandemly aligned in the genome. Lsdiaph1 and Lsdiaph2 encode diaphanous-related formin proteins composed of approximately 1100 amino acids. Proteins LsDiaph1 and LsDiaph2 have $89.4 \%$ identity at the amino acid sequence level ([16] Kuroda et al. 2016). Davidson et al. (2016) described the mutated gene as ldia2 (Lsdia2), while Kuroda et al. (2016) called it Lsdia1. Based on an assumption that the original (ancestral) one is molecularly more conserved than the duplicated counterpart, we here followed the naming of the gene by Davidson et al. (2016). 


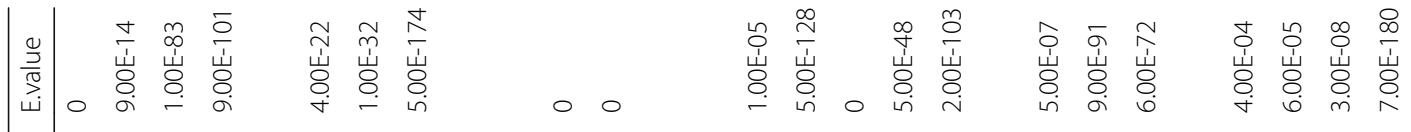

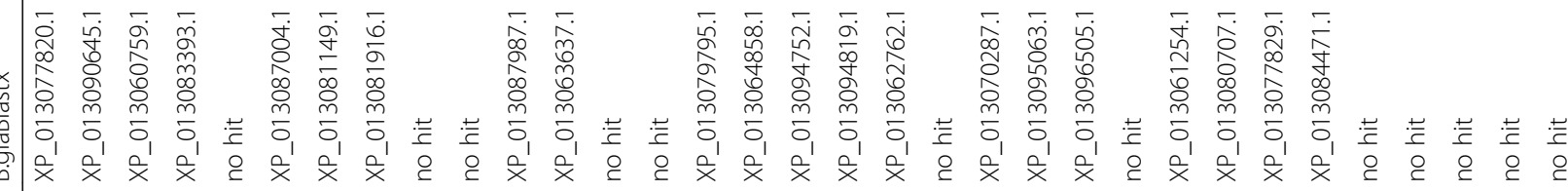

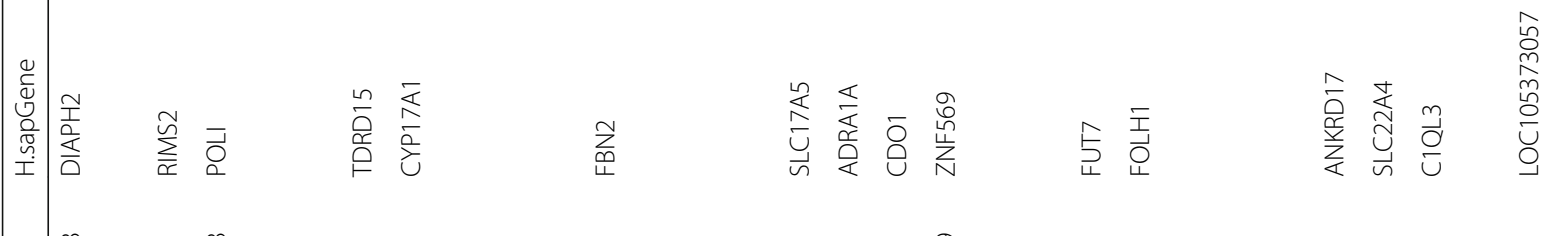

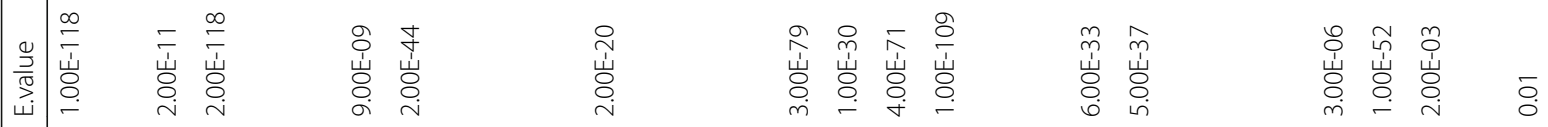

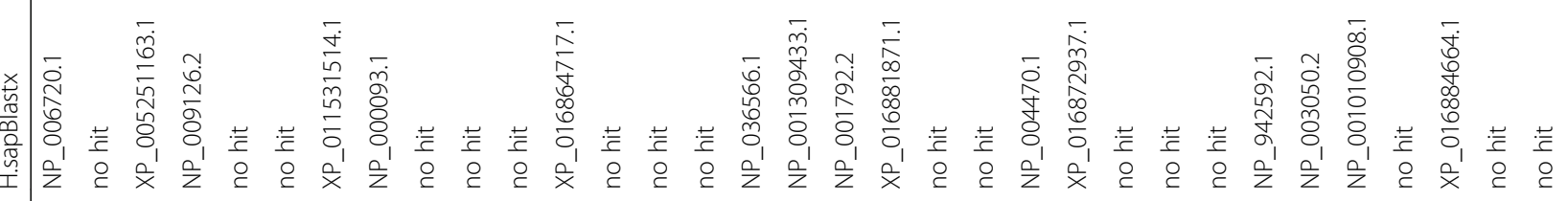

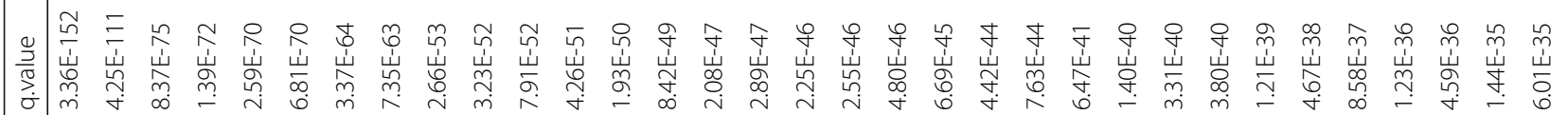

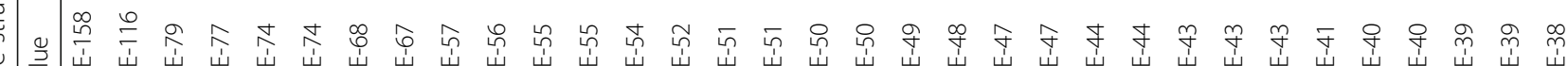
至

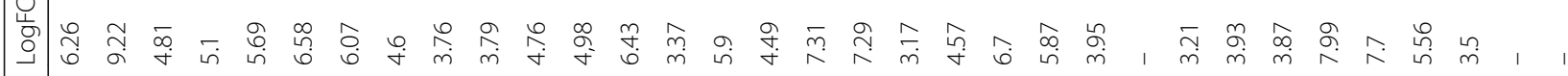

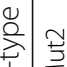

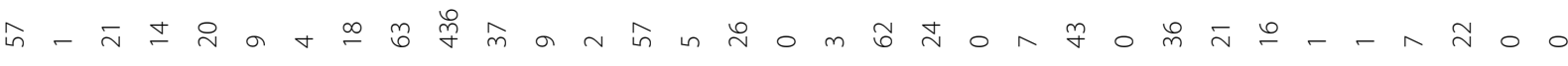

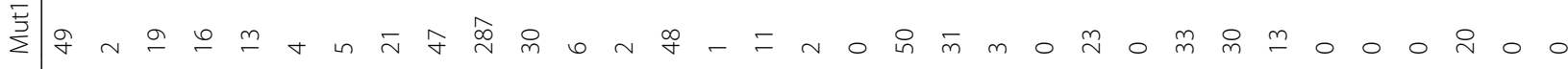

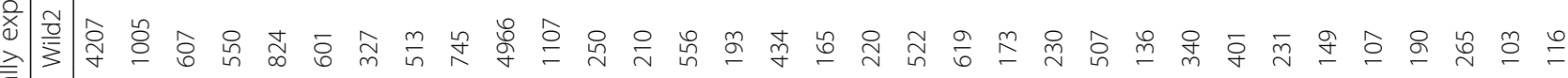

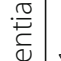

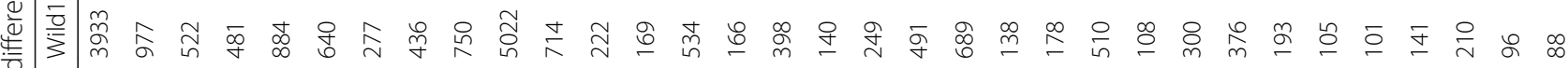

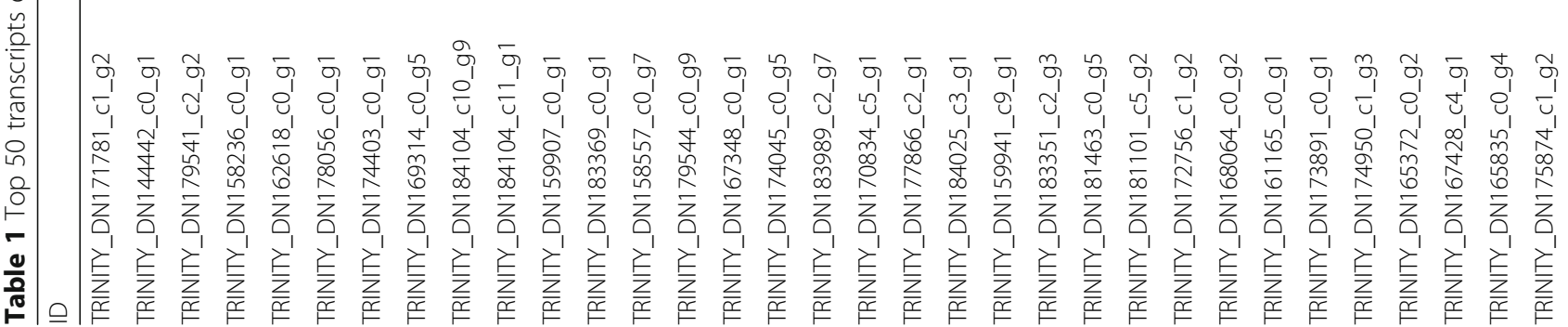




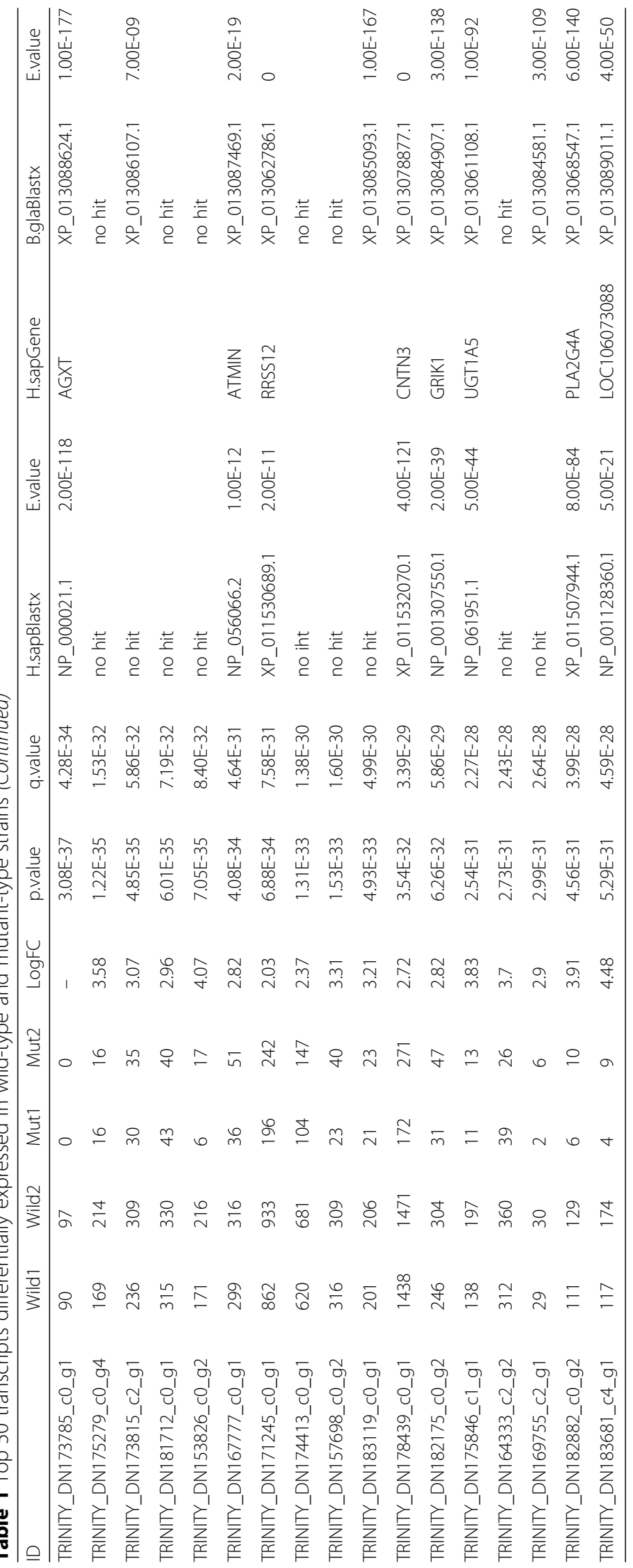


Two diaph genes in Lymnaea genome suggests that the $B$. similaris diaph gene is also duplicated, with the expression of one being downregulated. Therefore, we carefully examined maternal transcripts of Bsdiaph (diaph) in a wild strain and found two types of Bsdiaph transcript, between which we disntinguich as Bsdiaph-a (TRINITY_DN180102_c0_g1) and Bsdiaph-b (TRINITY_DN171781_c1_g2). Although their nucleic acid sequences were $71.9 \%$ conserved in their protein coding regions, expression of Bsdiaph-a didn't significantly differ between wild and mutant embryos through the EdgeR comparison ( $p$-value; 0.47). Molecular phylogenetic analysis indicated independent duplications of diaph genes in Lymnaea and Stylommatophora (Fig. 2).

Bsdiaph proteins have a Diaphanous GTPase binding domain (GBD), a Formin homology (FH3) domain, an FH2 domain, and a short Diaphanous auto-regulatory domain (DAD) ( $\mathrm{N}$ - to C-terminal). BsDiaph-a and BsDiaph-b share $70.0 \%$ amino acid sequence similarity (Fig. 3a).

In addition, we found splicing variants of Bsdiaph- $a$ in the maternal transcriptomes that encode two protein isoforms, Bsdiaph-a- $\times 1$ and Bsdiaph-a- $\times 2$ (Fig. 3a-C).
These two isoforms differ in their C-terminal amino acid sequences after the conserved DAD domain, Bsdiaph-a- $\times 2$ being shorter in the C-terminus compared with Bsdiaph-a- $\times 1$ (Fig. 3a). Bsidiaph-a-x 2 contained additional nucleotide sequences against Bsidiaph- $a-\times 1$ as in the case of these variants in B. glabrata and $A$. californica, and it caused frameshift and an earlier stop codon (Fig. 3c). Other variants which were found in the genome-sequenced gastropods, B. glabrata and A. californica, e.g. diaph $-\times 3$, were not found in our data sets.

\section{Expression of Bsdiaph- $a$ and Bsdiaph- $b$}

We identified a gene with two noteworthy features; (a) its maternal transcript level was greatly reduced in the mutant strain compared with the wild strain and (b) $B$. similaris expresses two different types of transcripts, Bsdiaph- $a$ and Bsdiaph-b, with the expression of the latter likely being down-regulated in the mutant. Therefore, we further examined expression profiles of the two genes by adding data obtained from juveniles and from adult digestive glands in both wild and mutant strains (Additional file 2).

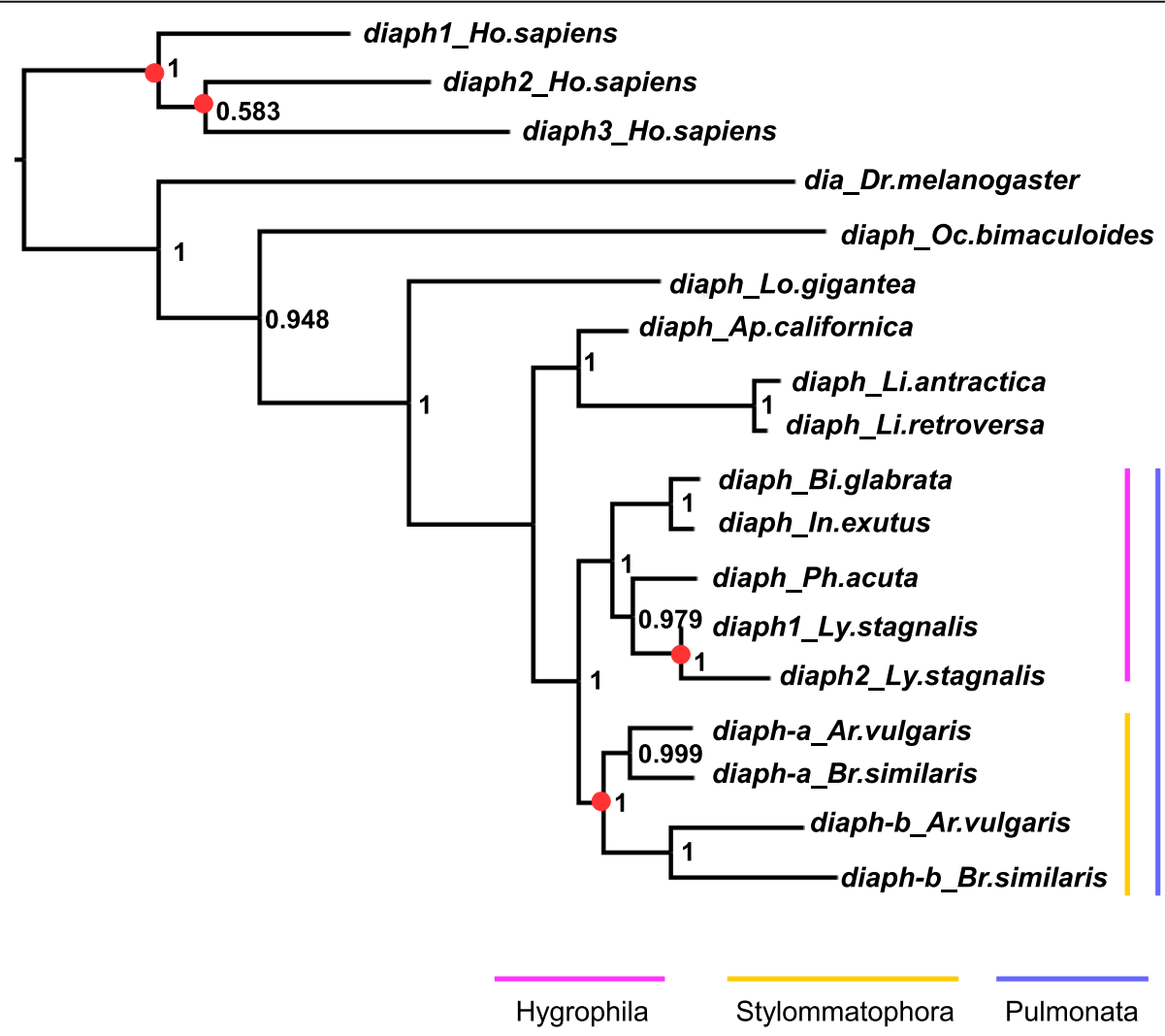

Fig. 2 Molecular phylogeny of diaph genes. Gene duplication was shown by red circles. Abbreviations are as follows. Homo sapiens; Ho.sapiens, Drosophila melanogaster; Dr.melanogaster, Octopus bimaculoides; Oc.bimaculoides, Lottia gigantea; Lo.gigantea, Aplysia californica; Ap.californica, Limacina antractica; Li. antractica, Limacina retroversa; Li.retroversa, Biomphalaria glabrata; Bi.glabrata, Indoplanorbis exustus; In.exustus, Physella acuta; Ph. acuta, Lymnaea stagnalis; Ly.stagnalis, Arion vulgaris; Ar.vulgaris, Bradybaena similaris; Br.similaris 


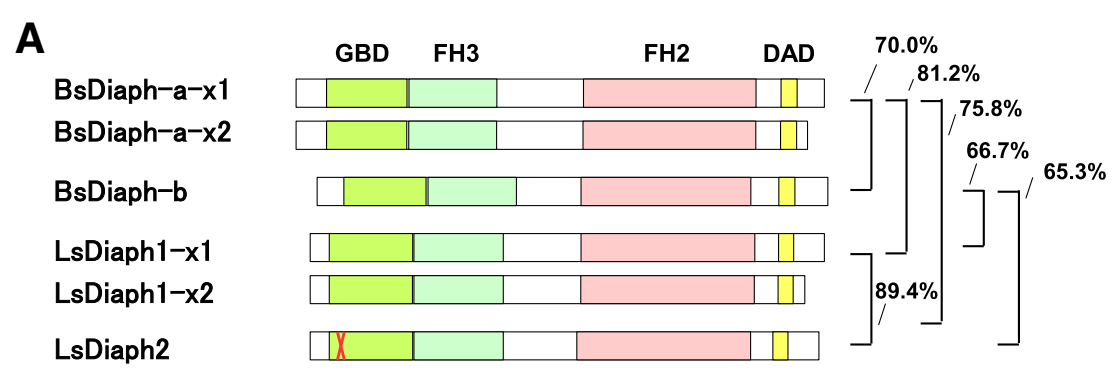

B
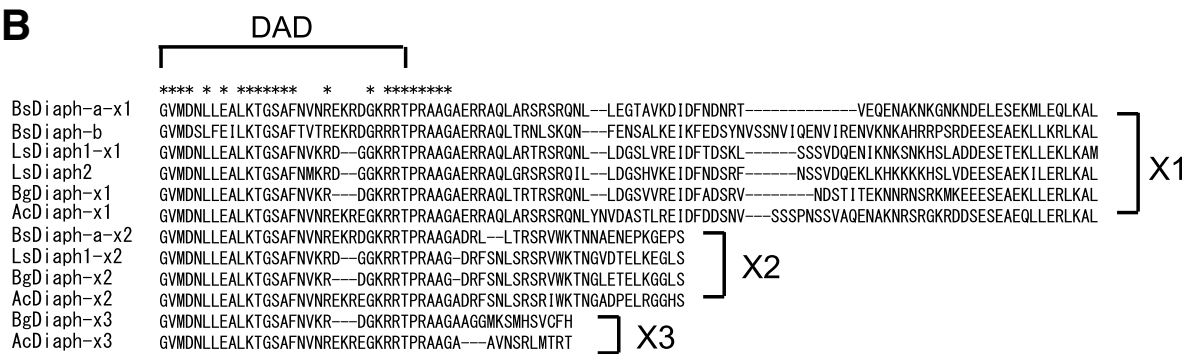
GVMONLLEALKTGSAFNUNREKREGKRRTPRAAGA--AVNSRLMTRT X

C

Bsdiaph-a-x1
Bsdiaph-b
Lsdiaph1-x1
Lsdiaph2
Bgdiaph-x1
Acdiaph- $x 1$
Bsdiaph-a-x2
Lsdiaph1-x2
Bgdiaph- $x 2$
Acdiaph- $x 2$
Bgdiaph- $x 3$
Acdiaph $-x 3$

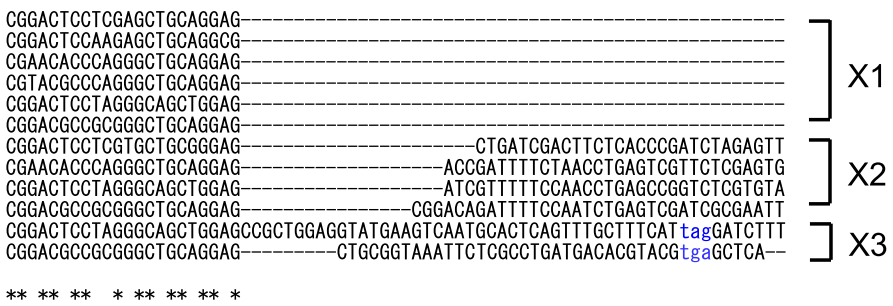

Bsdiaph-a-x1

Bsdiaph-a-
Bsdiaph- $b$

Lsdiaph-b
Lsdiaph1-x1
Lsdiaph2

Bgdiaph-x1

Bgdiaph $-x 1$
Acdiaph $-x 1$

Acdiaph-x1

Bsdiaph $-a-x 2$
Lsdiaph1 $-x 2$

Lsdiaph1-x2
Bgdiaph $-x 2$

Bgdiaph $-x 2$
Acdiaph $-x 2$

Acdiaph- $x 2$
Bgdiaph $-x 3$

Acdiaph $-\times 3$

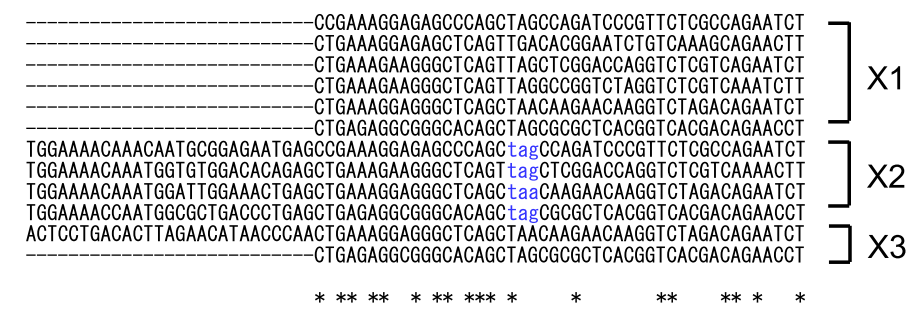

Fig. 3 Diaph protein structures. (a) Those translated by Bsdiaph-a (two splicing variants, $\times 1$ and $\times 2$ ) and Bsdiaph-b. A truncated mutation of LsDiaph-1 is also shown with a red X (from Kuroda et al. 2016). Diaph GBD; Diaphanous GTPase binding domain FH3; Diaphanous FH3 domain FH2; FH2 domain DAD; Diaphanous auto-regulatory domain. (b) Comparison of C-terminal amino acid sequences of Diaph between various snail species. (c) Alignments of splice variants of diaph. Stop codons are shown by blue lower-case letters. Bs, Bradybaena similaris, Ls, Lymnaea stagnalis, Bg, Biomphalaria glabrata, Ac, Aplysia californica

The TPM (tags per million) score of Bsdiaph-a mRNA was comparable between wild and mutant strains, although slightly lower in the mutant in early-stage embryos (nearly $80 \%$ of wild strain) (Fig. 4a). Because Bsdiaph-a mRNA is a maternal transcript, its TPM score decreased in juveniles and in adult digestive gland. In contrast, the TPM score of Bsdiaph-b differed considerably between wild and mutant strains. The number of Bsdiaph- $b$ transcripts in early mutant embryos was less than $1 \%$ that of the wild strain (Fig. 4b). The Bsdiaph-b transcripts was few in number in juveniles and adult digestive glands of both strains. These results indicate that the expression of Bsdiaph-b is maternally suppressed in mutants. On the other hand, maternal expression of Bsdiaph- $a$ is normally regulated in both wild and mutant strains. This result was also validated by quantification through qRT-PCR methods. Figure 4c shows expression level of Bsdiaph- $a$ and Bsdiaph-b from early embryos of wild, racemic and F1 strains with triplicate biological samples through qRT-PCR. Relative expression level of Bsdiaph-a was comparable among three strains as the result of quantification by RNA-Seq data. 
A

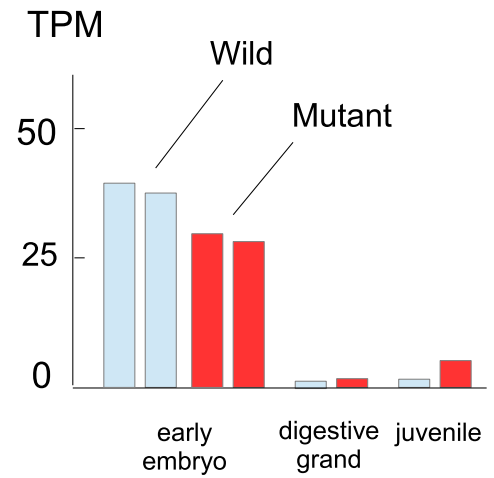

diaph-a

C

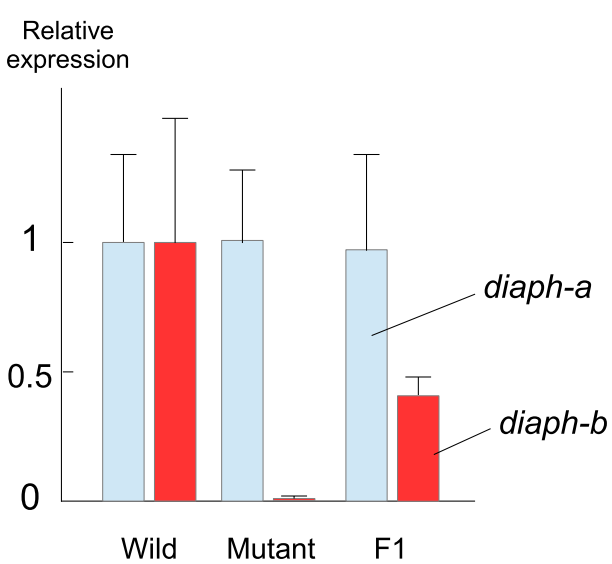

B

TPM

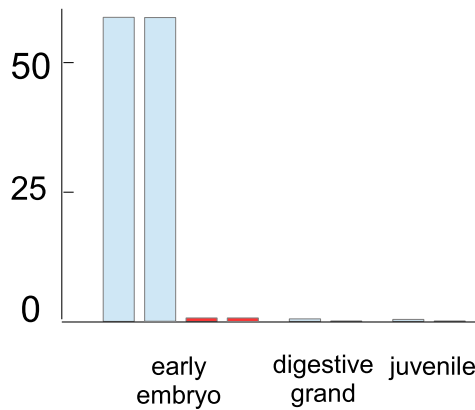

diaph-b early embryos, juveniles, and digestive glands, based on mRNA quantity (TPM, tags per million). Blue bars indicate the TPM score of the wild-type and red bars denote that of the mutant strain. (c) Quantification of Bsdiaph- $a$ and Bsdiaph-b in the early embryos from wild, mutant and F1 strains by qRT-PCR $(n=3)$. Standard error was shown by error bars

On hand expression of Bsdiaph-b from embryos of the racemic mutant strain was highly reduced and those from F1 strain was lower than those from the wild strain.

\section{Bsdiaph- $b$ transcript is not frame-shifted}

In sinistral mutant strain of Lymnaea stagnalis, Lsdiaph2 transcribes mRNA in which a single-base deletion occurs at position 50 of the coding region, which causes a frame-shift to produce truncated diaphanous protein consisting of only the N-terminus (Fig. 3a). To determine whether this is the case in Bsdiaph-b, we carefully examined maternal transcripts of Bsdiaph- $b$ from mutant embryos, juvenile, and adult digestive gland (Table 1).

Although the central region (55 nucleotides) of Bsdiaph- $b$ transcripts showed a few substitutions of nucleotides (Fig. 5), no clear lesions were identified in BsDiaph-b proteins. We found no non-sense mutations, including frame-shifted transcripts that produce truncated proteins. Instead, at least two alleles were found in the reads from a mutant strain (Fig. 5). These alleles were not from sequencing errors because the reads from four independent samples (early-embryo1, early-embryo2, digestive gland and juvenile) contained two alleles. As the racemic strain of $B$. similaris should be homozygotic for the mutated locus ([24] Utsuno and Asami, 2010), we concluded that Bsdiaph- $b$ is not mutated.

\section{Discussion}

The present RNA-seq analysis of differential expression of maternal transcripts in wild (dextral cleavage) and mutant strains (dextral or sinistral cleavage) of Bradybaena similaris showed that (1) Bsdiaph has two types of transcripts, suggesting the presence of two copies of the gene (Bsdiaph- $a$ and Bsdiaph- $b$ ) in the genome; (2) the amount of maternal mRNA of Bsdiaph- $a$ was comparable between the two strains, but that of Bsdiaph- $b$ was highly reduced in mutant eggs, suggesting that suppression of Bsdiaph-b expression is involved in the mutation; (3) the Bsdiaph-b transcript is not frame-shifted 


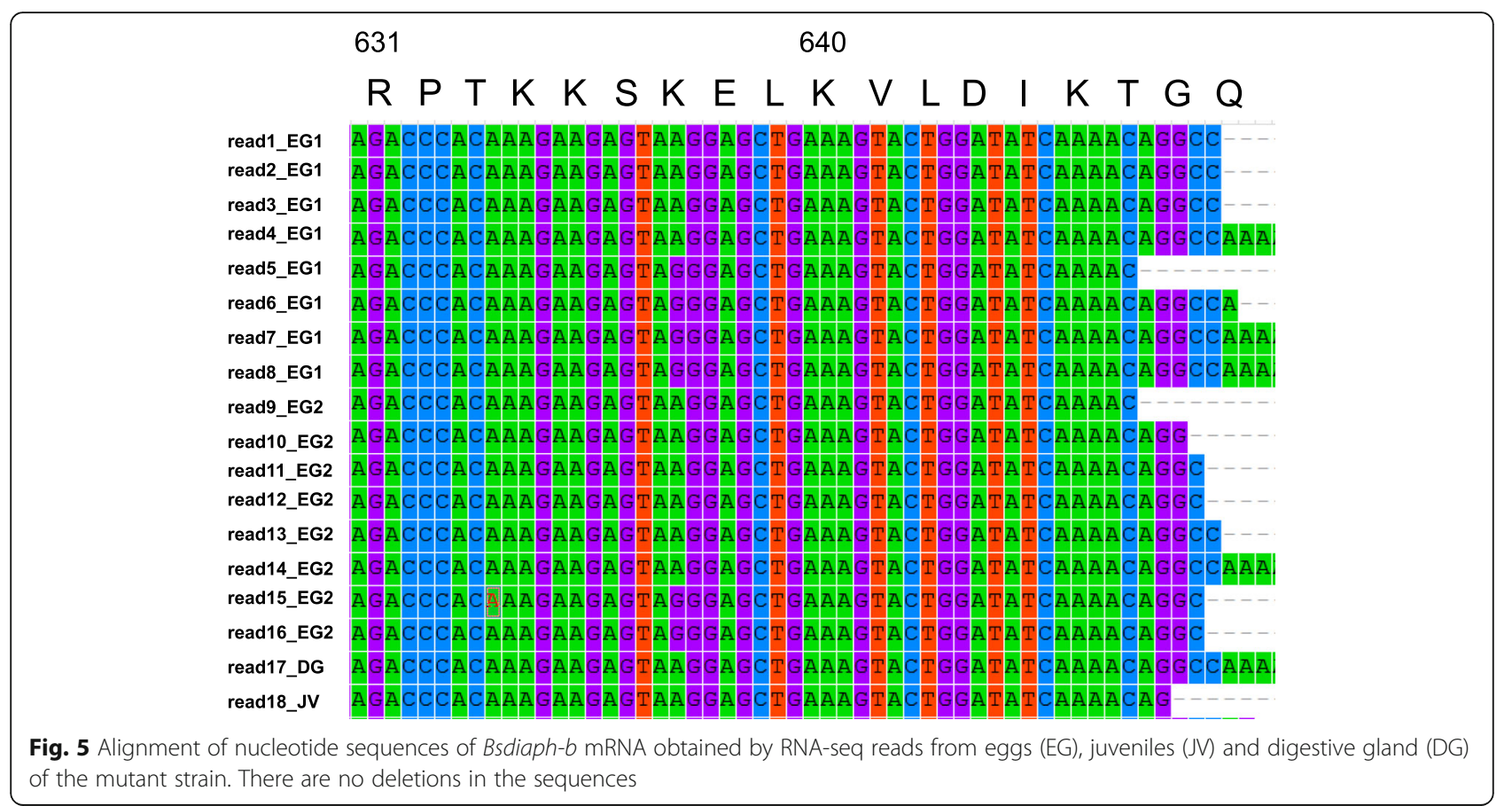

in mutant eggs, suggesting that the gene itself is normal. Our finding of no mutation at Bsdiaph- $b$, which is only weakly expressed in the progeny of the racemic mutant, suggests the presence of some regulatory mechanism that is responsible for polarity determination for viable spiral cleavage, other than duplicated diaph homologs. The mutated gene may be an upstream trans-regulator or a distantly located cis-element from the locus of Bsdiaph- $b$ because of heterogeneity of the sequence of Bsdiph- $b$ from mutant snails.

Lymnaea stagnalis duplicated diaph into tandem -aligned Lsdiaph1 and Lsdiaph2. Diaph protein may have pivotal roles in coordinating functions that depend upon actin filaments ([14] Shibazaki et al., 2004). Davison et al. [16] showed that Lsdiaph1 and Lsdiaph2 $m R N A$ are distributed unevenly into one of the cells in the 2-cell embryo (dextral), while Kuroda et al. [17] found an even distribution. This apparent contradiction probably results from the aberrant variability of chiral patterning among mostly non-viable embryos in the Lymnaea mutant strain ([12] Utsuno et al. 2011). Lsdiaph2 mutated to form a functionless protein, so that in the homozygotic ss mutant, a reduced amount of Diaph protein is produced. If diaph were a single-copy gene with a critical role in early embryonic cleavage, its mutation might disturb the cleavage pattern, resulting in abnormal embryos. Embryonic lethality of mutant animals with a single copy of diaph in Drosophila melanogaster and Caenorhabditis elegans, supports this idea ([32] Castrillon and Wasserman, 1994, [33] Swan et al., 1998). On the other hand, when the gene becomes duplicated and one of the two copies mutates, spiral cleavage may proceed enabling viable embryogenesis. However, only a small portion of siblings survive to hatch as the sinistral in this case of the Lymnaea mutant ([24] Utsuno and Asami, 2010).

Diaph in Bradybaena similaris is also duplicated. Our expression analysis indicated reduced expression of $B s d i a p h-b$ and normal expression of Bsdiaph- $a$ in early embryos of the mutant strain. This transcriptional phenotype is similar to the Lymnaea mutant. Although the differential expression of Bsdiaph- $a$ and Bsdiaph- $b$ implies their diversification, products from Bsdiaph- $a$ could compensate for loss of crucial functions of Diaph protein in early embryogenesis when Bsdiaph-b expression is reduced.

Despite their similarity in diaph homologs' expression patterns, the mutant phenotypes differ between $L$. stagnalis and B. similaris. The racemic mutant in B. similaris generates both dextral and sinistral progeny, while offspring of the Lymnaea mutant develop into the sinistral only, although the majority of siblings fail in early embryogenesis in both cases. One possible explanation for these phenotypes is gleaned from the difference between null and leaky mutants. Lsdiaph2 of the Lymnaea mutant strain is frame-shifted and is considered a null mutation, while no clear lesions were found in the sequence of Bsdiaph- $b$. A slight amount of Bsdiaph- $b$ was detected in early embryos from the racemic mutant of $B$. similaris, even though expression was highly reduced (Fig. 4). Although our RNA-seq data doesn't establish absence or presence of proteins, it is possible that the 
reduced amount of the gene product from Bsdiaph- $b$ is produced in racemic mutant embryos, and it partly compensates for their phenotypes. If the gene product of $B s$ diaph- $b$ is crucial for the change of cleavage direction from sinistral to dextral, and the racemic mutant of $B$. similaris is a leaky mutant, their siblings become mixture of both dextral and sinistral individuals.

Another hypothesis is that the default chiral directionality without functional protein products of duplicated diaph differs between the dextral Lymnaeidae clade that relatively recently evolved by reversal from the sinistral ancestor in the Hygrophila ([16] Davison et al. 2016) and the other Baradybaena clade of the dextral family Camaenidae that has long retained dextrality in the Stylommatophora ([34] Köhler and Criscione, 2015). In L. stagnalis, spiral cleavage becomes somewhat reversed, and a fraction of sinistrally developing siblings survive to hatching. In B. similaris, on the other hand, no clear direction could be determined for spiral cleavage. Early embryonic cells could perform sinistral cleavage by lacking a determinant to be dextral in Lymnaea mutant, while the defect of polarity regulation in embryonic cells results in directionally randomized cleavage in the Bradybaena mutant (Fig. 1b).

Recent reports have highlighted the importance of cellular chirality for determination of left-right handedness of several organs during embryogenesis ([35] Taniguchi et al., 2011, [36] Inaki et al., 2016). In Drosophila melanogaster, embryonic gut, male genitalia and adult hind gut are directionally rotated organs, and their proper morphogenesis to right or left is controlled by the chirality of each cell. One of the functions of formin proteins is control of cellular chirality through construction of radial fibers of actin filaments ([37] Tee et al., 2016). Actin filaments are important for spiral cleavage ([13] Shibazaki et al., 2004). Between dextral Lymnaea and sinistral Physa, early blastomeres are reversed in cellular chirality. The cortical layer of blastomere even at the first cell division moves clockwise in the dextral group but counterclockwise in the sinistral group ([38] Meshcheryakov and Beloussov, 1974). Brun-Usan et al. [39] pointed out the importance of directed cellular chirality for proper spiral cleavage by computational simulation. The spiral cleavage of progeny from the racemic mutant of $B$. similaris resembles their direction-randomized spiral cleavage with no directed cortical rotation.

In gastropods that copulate for reproduction, left-right reversal results in genital mismatch with the wild type because the genital orifix is located in the body side instead of the midline. Thus, population fixation for reversal contributes to reproductive isolation, especially in stylommatophoran pulmonates that copulate simultaneously reciprocally ([40] Gittenberger, 1988; [23] Asami et al., 1998; [20] Ueshima and Asami, 2003).
Left-right reversal also functions for surviving or avoiding predation in some environments ([41] Hoso et al., 2007, [21] Hoso et al., 2010, [42] Danaisawadi et al., 2016). Southeast Asian snail-eating snakes specialize in predation on the dextral majority in snails and avoid or fail to capture sinistrals. The advantage of sinistrality for avoidance of predation accelerated speciation through left-right reversal from dextral ancestors in the range of these snakes. In such cases, left-right reversal by a single gene may have played a critical role for speciation. However, molecular genetic mechanisms for their developmental reversal have not been identified. Left-right reversal of spiral cleavage via mutation or transcriptional reduction of one diaph might have been involved for the evolution of reversed populations/species. If the presence of two diaph genes by duplication might have been advantageous for evolution of the reversal in pulmonate snails, this should be considered an exaptation.

The evolution of left-right reversal requires population fixation for reversal that permits equivalent or superior Darwinian fitness relative to the wild type ([18] Okumura et al., 2008). However, in either cases of the mutants of L. stagnalis ([11] Asami et al. 2008, [12] Utsuno et al. 2011) or B. similaris ([24] Utsuno and Asami, 2010), most of sibling embryos exhibit aberrant reversals and fail to hatch. Thus, these mutations are to be eliminated by internal selection and cannot give rise to sinistral populations or species. In contrast, for example, the wild-type sinistral embryos of the hygrophilan pond snails Physa and Biompharalia typically perform reversed mirror-image cleavage compared with the wild-type dextral cleavage of Lymnaea ([43] Crampton, 1894, [44] Camey and Verdonk, 1970) unlike the "sinistral" mutant of $L$. stagnalis. This means that we do not yet know about mutations and genetic mechanisms that could have generated reversed snail species from the dextral or sinistral ancestral populations. Our study accordingly has important implications to explore how reversed populations/species have recurrently evolved if mutation for the diaph function has been involved.

\section{Conclusions}

Maternal expression of one of the diaph homolog $($ Bsdiaph $-b)$ is most reduced in progeny of the racemic mutant of the stylommatophoran pulmonate Bradybaena similaris, which generates both dextral and sinistral progeny. The other diaph homolog (Bsdiaph-a) is equivalently expressed between the wild-type dextral and mutant. These homologs originated by duplication in the stylommatophoran ancestor. However, no clear lesion is present in the diaph gene itself, unlike the case of frame-shift mutation which results in incomplete sinistral development in the hygrophilan pulmonate Lymnaea stagnalis. Our results suggest that a regulatory 
mechanism controls the left-right polarity of spiral cleavage. Our study provides a new molecular basis that promotes further studies for understanding of the evolution of spiral cleavage in gastropods which frequently evolved left-right reversal.

\section{Additional files}

Additional file 1: Table S2. IDs of genes for the molecular phylogenetic analysis. (DOC $20 \mathrm{~kb}$ )

Additional file 2: Table S1. RNA-seq reads by Hiseq4000 of Bradybaena similaris. (XLS $9 \mathrm{~kb}$ )

\section{Acknowledgements}

We thank Masatoyo Okamoto for his collection of the sinistral mutant of $B$. similaris, Satoko Kuwabara for her maintenance of its strains, Hiroki Utsuno for providing several images and Steven D. Aird for his help in preparation of the manuscript. We also thank the DNA Sequence Section at OIST for sequencing, and we are grateful to Kanako Hisata for primary processing of sequencing data.

\section{Funding}

The work was supported in part by JSPS grants to TA (17H01673, 17H01448), and OIST Internal Research Fund to NS (Marine Genomics Unit).

\section{Availability of data and materials}

All data generated or analyzed during this study are deposited into DDBJ databank (\#00000000)

\section{Authors' contributions}

TN, NS and TA conceived the project and TN performed analyses. TN, NS and TA prepared the manuscript and approved the final submission.

\section{Ethics approval and consent to participate}

Not applicable.

\section{Consent for publication}

Not applicable.

\section{Competing interests}

The authors declare that they have no competing interests.

\section{Publisher's Note}

Springer Nature remains neutral with regard to jurisdictional claims in published maps and institutional affiliations.

\section{Author details}

'Department of Biology, Faculty of Sciences, Shinshu University, Matsumoto 390-8621, Japan. ${ }^{2}$ Marine Genomics Unit, Okinawa Institute of Science and Technology Graduate University, Onna, Okinawa 904-0495, Japan.

Received: 19 August 2018 Accepted: 26 December 2018

Published online: 10 January 2019

\section{References}

1. Gilbert SF, Raunio AM. Embryology: Constructing the organism. Sunderland, MA: Sinauer Associates; 1997.

2. Nielsen C. Animal evolution. 3rd ed. Oxford: Oxford Uni. Press; 2012.

3. Dunn CW, Hejnol A, Matus DQ, Pang K, Browne WE, et al. Broad phylogenomic sampling improves resolution of the animal tree of life. Nature. 2008;452:745-9.

4. Henry JQ. Spiralian model systems. Int J Dev Biol. 2014;58:389-401.

5. Laumer CE, Bekkouche N, Kerbl A, Goetz F, Neves RC, et al. Spiralian phylogeny informs the evolution of microscopic lineages. Curr Biol. 2015;25: 2000-6.
6. Kuroda R, Endo B, Abe M, Shimizu M. Chiral blastomeres arrangement dictates zygotic left-right asymmetry pathway in snails. Nature. 2009;462: 790-4.

7. Toyama K. Maternal inheritance and mendelism. J Genet. 1913;2:351-405.

8. Boycott $A E$, Diver C. On the inheritance of sinistrality in Limnaea peregra. Proc R Soc Biol Sci Ser B. 1923:95:207-13.

9. Sturtevant AH. Inheritance of direction of coiling in Limnaea. Science. 1923; 58:269-70.

10. Freeman G, Lundelius JW. The developmental genetics of dextrality and sinistrality in the gastropod Lymnaea peregra. Roux Arch Dev Biol. 1982;191: 69-83.

11. Asami T, Gittenberger E, Falkner G. Whole-body enantiomorphy and maternal inheritance of chiral reversal in the pond snail Lymnaea stagnalis. J Hered. 2008;99:552-7.

12. Utsuno $H$, Asami T, Dooren TJMV, Gittenberger E. Internal selection against the evolution of left-right reversal. Evolution. 2011;65:2399-411.

13. Hierck BP, Witte B, Poelmann RE, Gittenberger-de Groot AC, Gittenberger E. Chirality in snails is determined by highly conserved asymmetry genes. J Mollus Stud. 2005;71:192-5.

14. Shibazaki Y, Shimizu M, Kuroda R. Body handedness is directed by genetically determined cytoskeletal dynamics in the early embryo. Curr Biol. 2004:24:1462-7.

15. Harada $Y$, Hosoiri $Y$, Kuroda R. Isolation and evaluation of dextral-specific and dextral-enriched CDNA clones as candidates for the handednessdetermining gene in a freshwater gastropod, Lymnaea stagnalis. Dev Genes Evol. 2004;214:159-69.

16. Davison A, McDowell GS, Holden JM, Johnson HF, Koutsovoulos GD, et al. Formin is associated with left-right asymmetry in the pond snail and the frog. Curr Biol. 2016;26:654-60.

17. Kuroda R, Fujikura K, Abe M, Hosoiri Y, Asakawa S, et al. Diaphanous gene mutation affects spiral cleavage and chirality in snails. Sci Rep. 2016;6:34809.

18. Okumura T, Utsuno H, Kuroda J, Gittenberger E, Asami T, Matsuno K. The development and evolution of left-right asymmetry in invertebrates: lessons from Drosophila and snails. Dev Dyn. 2008:237:3497-515.

19. Gittenberger E, Hamann TD, Asami T. Chiral speciation in terrestrial pulmonate snails. PLoS One. 2012;7:e34005.

20. Ueshima R, Asami T. Single-gene speciation by left-right reversal. Nature. 2003;425:679.

21. Hoso M, Kameda Y, Wu SP, Asami T, Kato M, Hori M. A speciation gene for left-right reversal in snails results in anti-predator adaptation. Nat Commun. 2010;1:133.

22. Kornilios P, Stamataki E, Giokas S. Multiple reversals of chirality in the land snail genus Albinaria (Gastropoda, Clausiliidae). Zool Scr. 2015;44:603-11.

23. Asami T, Ohbayashi $\mathrm{K}$, Cowie RH. Evolution of mirror images by sexually asymmetric mating behaviour in hermaphroditic snails. Am Nat. 1998;152: 225-36.

24. Utsuno $\mathrm{H}$, Asami T. Maternal inheritance of racemism in the terrestrial snail Bradybaena similaris. J Hered. 2010;101:11-9.

25. van den Biggelaar JAM. RNA synthesis during cleavage of the Lymnaea egg Exp Cell Res. 1971;67:207-10.

26. Grabherr MG, Haas BJ, Yassour M, Levin JZ, Thompson DA, et al. Full-length transcriptome assembly from RNA-Seq data without a reference genome. Nat Biotechnol. 2011;29:644-52.

27. Haas BJ, Papanicolaou A, Yassour M, Grabherr M, Blood PD. De novo transcript sequence reconstruction from RNA-seq using the trinity platform for reference generation and analysis. Nat Protoc. 2013;8:1494-512.

28. Trapnell C, Roberts A, Goff L, Pertea G, Kim D, et al. Differential gene and transcript expression analysis of RNA-seq experiments with TopHat and cufflinks. Nat Protoc. 2012;7:562-78.

29. Robinson MD, McCarthy DJ, Smyth GK. edgeR: a Bioconductor package for differential expression analysis of digital gene expression data. Bioinformatics. 2010;26:139-40.

30. Noda T. The maternal genes Ci-p53/p73- $a$ and Ci-p53/p73-b regulate zygotic ZicL expression and notochord differentiation in Ciona intestinalis embryos. Dev Biol. 2011;360(1):216-29.

31. McCarthy JD, Chen Y, Smyth GK. Differential expression analysis of multifactor RNA-Seq experiments with respect to biological variation. Nucleic Acids Res. 2012:40:4288-97.

32. Castrillon DH, Wasserman SA. Diaphanous is required for cytokinesis in Drosophila and shares domains of similarity with the products of the limb deformity gene. Development. 1994;120:3367-77. 
33. Swan KA, Severson AF, Carter JC, Martin PR, Schnabel H. Cyk-1: a C. elegans FH gene required for a late step in embryonic cytokinesis. J Cell Sci. 1998; 111:2017-27.

34. Köhler F, Criscione F. A molecular phylogeny of camaenid land snails from North-Western Australia unravels widespread homoplasy in morphological characters (Gastropoda, Helicoidea). Mol Phylogenet Evol. 2015;83:44-55.

35. Taniguchi K, Maeda R, Ando T, Okumura T, Nakazawa N, Hatori R, Nakamura M, Hozumi S, Fujiwara H, Matsuno K. Chirality in planar cell shape contributes to left-right asymmetric epithelial morphogenesis. Science 2011; 15;333(6040):339-341.

36. Inaki M, Liu J, Matsuno K. Cell chirality: its origin and roles in left-right asymmetric development. Philos Trans R Soc Lond Ser B Biol Sci. 2016;19: 371(1710).

37. Tee YH, Shemesh T, Thiagarajan V, Hariadi RF, Anderson KL, Page C, Volkmann N, Hanein D, Sivaramakrishnan S, Kozlov MM, Bershadsky AD. Cellular chirality arising from the self-organization of the actin cytoskeleton. Nat Cell Biol. 2015;17:445-57.

38. Meshcheryakov VN, Beloussov LV. Asymmetrical rotations of Blastomeres in early cleavage of Gastropoda. Wilhelm Roux's Archives. 1975;177:193-203.

39. Brun-Usan M, Marín-Riera M, Grande C, Truchado-Garcia M, Salazar-Ciudad I. A set of simple cell processes is sufficient to model spiral cleavage. Development. 2017:144:54-62.

40. Gittenberger T. Sympatric speciation in snails; a largely neglected model. Evolution. 1988:42:826-8.

41. Hoso M, Asami T, Hori M. Right-handed snakes: convergent evolution of asymmetry for functional specialization. Biol Lett. 2007:3:169-73.

42. Danaisawadi $P$, Asami T, Ota H, Sutcharit C, Panha S. A snail-eating snake recognizes prey handedness. Sci Rep. 2016;6:23832.

43. Crampton HE. Reversal of cleavage in a sinistral gastropod. Ann N Y Acad Sci. 1894:8:167-9.

44. Camey T, Verdonk N. The early development of the snail Biomphalaria glabrata (say) and the origin of the head organs. Neth J Zool. 1970;20:93-121.

Ready to submit your research? Choose BMC and benefit from:

- fast, convenient online submission

- thorough peer review by experienced researchers in your field

- rapid publication on acceptance

- support for research data, including large and complex data types

- gold Open Access which fosters wider collaboration and increased citations

- maximum visibility for your research: over $100 \mathrm{M}$ website views per year

At $\mathrm{BMC}$, research is always in progress.

Learn more biomedcentral.com/submissions 